\title{
Full-Wave Analysis of Nonplanar Transmission Lines on Layered Medium by Means of MPIE and Complex Image Theory
}

\author{
Joaquin Bernal, Francisco Medina, Member, IEEE, and Rafael R. Boix, Member, IEEE
}

\begin{abstract}
In this paper, a multiconductor transmission line consisting of arbitrary cross-sectional perfect conductors printed on a layered isotropic or uniaxial anisotropic dielectric medium is analyzed by solving the mixed-potential integral equation for the free-surface currents. Closed-form expressions of the two-dimensional space-domain Green's functions for the electrodynamic potentials are used. These expressions are obtained by applying the complex image technique to the spectral functions remaining after removing the asymptotic and pole contributions from the original Green's functions. A single set of complex images is obtained for any guess value of the unknown propagation constant and for any pair of source/field points. In addition, the reaction integrals involved in the application of the method of moments are worked out in a quasi-analytical way. The final result is an accurate and highly efficient computation code for analyzing multiconductor structures printed on a layered medium.
\end{abstract}

Index Terms-Complex image method, integral equations, layered media, nonplanar transmission lines.

\section{INTRODUCTION}

$\mathbf{T}$ HE analysis of multiconductor transmission lines printed on layered substrates has been a popular research topic for a long time due to the importance of this physical system in various practical applications, including microwave integrated circuits, high-speed interconnects [1], and discrete wire technology [2]. Highly efficient and accurate analysis tools have been developed for the particular and important case of planar conductors both under quasi-TEM approximation [3] and rigorous full-wave analysis [4]. However, nonplanar conductors must be considered in many practical situations. Rectangular and trapezoidal cross sections should be considered, for instance, in transmission lines appearing in monolithic integrated circuits or high-speed interconnects. Tightly coupled strips must be analyzed by considering their nonzero thickness. In general, a computer code capable of analyzing transmission lines having general cross-sectional conductors may find many applications. Such a code can be developed on the basis of purely numerical methods such as finite elements [5] or finite differences [6]. Other techniques, such as the mode-matching method [7], [8] or the method of lines [9] can be applied

Manuscript received October 28, 1999. This work was supported by the Comisión Interministerial de Ciencia y Technología under Project TIC98-0630.

J. Bernal is with the Department of Applied Physics, University of Seville, 41092 Seville, Spain.

F. Medina and R. R. Boix are with the Microwave Group, Department of Electronics and Electromagnetism, University of Seville, 41012 Seville, Spain (e-mail: medina@cica.es).

Publisher Item Identifier S 0018-9480(01)00015-1. to some particular structures. However, if suitable Green's functions are available, integral-equation methods are expected to be more accurate and less time consuming than any other method. This is the case for conductors embedded in layered media, for which spectral-domain Green's functions can be obtained in closed form. In this way, Michalski et al. analyzed transmission lines with arbitrary cross-sectional conductors on a single-layer substrate by using the mixed-potential integral equation (MPIE) formulation in conjunction with the method of moments [10]. In that paper, the spectral-domain Green's functions were transformed into the space domain by means of numerical integration. Later on, different formulations of the spectral-domain approach have been employed for studying the influence of the metallization thickness on single and coupled strip lines and microstrip structures [11]-[13]. These papers consider parallel conductors of rectangular shape. The integral-equation method in space and spectral domains has also been successfully employed for investigating the propagation in transmission lines of more complicated conductor cross sections embedded in multilayer substrates [14], [15].

In this paper, a fast and accurate technique is proposed for analyzing open arbitrary cross-sectional multiconductor lines printed on a layered substrate. This structure is shown in Fig. 1. The dielectric substrate consists of $N_{l}$ dielectric layers, which may be uniaxially anisotropic (having a $z$-directed optical axis). The method in this paper starts from the mixed-potential integral-equation (MPIE) formulation in the space domain [16], [17]-[19]. The key contribution of our approach lies on the way of obtaining the kernel of the integral equation: a very accurate approximation of the two-dimensional (2-D) space-domain Green's functions for the scalar and vector potentials is obtained for any source/field point pair in closed form. To accomplish this, the complex image method is employed. This method was proposed in [20] to carry out the closed-form evaluation of the Sommerfeld integrals arising from the analysis of radiating objects embedded in a layered medium. The complex image concept has been applied to transmission-line problems in the frame of the quasi-TEM approach [3]. Very recently, it has also been used by the authors for the full-wave analysis of planar transmission lines [21], [22]. In this paper, we propose to apply complex images to the analysis of nonplanar lines. The straightforward application of this technique gives place to serious problems. In order to overcome such difficulties, we propose to use the complex image method for approximating the Green's functions once the contributions of the asymptotic behavior and the surface-wave poles of the spectral Green's functions have been 


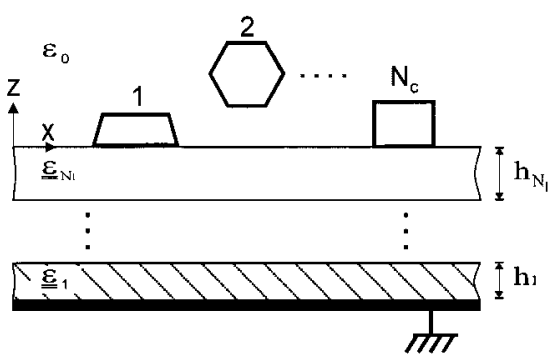

Fig. 1. Cross section of the structure under analysis. $N_{c}$ conductors of arbitrary cross section above a grounded multilayer dielectric slab.

extracted out. In this way, a single set of images is enough to provide a very accurate description of the Green's functions for any pair of source/field points and for any guess value of the unknown complex propagation constant. Therefore, the repeated evaluation of the Green's functions for different source and field points combinations and for different values of the propagation constant (eigenvalue search process) is performed in a very fast way. It is demonstrated in this paper that if the surface-wave pole contribution is meaningful and is not extracted out, complex images provide a poor approximation of the Green's function. Due to this reason, this paper provides an efficient quasi-analytical method for computing the surface-wave contribution to the space-domain kernel of the integral equation. This equation is finally solved by means of the method of moments. It must be pointed out that the contribution of the logarithmic singularity of the kernel to the moment-method integrals has been analytically evaluated so as to keep a high overall numerical efficiency.

\section{Formulation of the Problem}

Let us consider the open multiconductor system shown in Fig. 1, which consists of $N_{c}$ perfect electric conductors of arbitrary cross section placed above a stratified substrate made of $N_{l}$ dielectric layers. The layers are lossless isotropic or uniaxial anisotropic dielectrics with $z$-directed optical axis and they are assumed to be of infinite extent along the $x$-direction. The stratified substrate is coated by an infinite perfect conductor ground plane. Since we are interested in modes propagating along the $y$-direction, we assume a common phase factor $e^{-j \beta y}$ for fields and currents, $\beta$ being the unknown propagation constant. By enforcing the boundary condition for the tangential electric field at the surface of the conductors, we obtain an electric-field integral equation (EFIE). The kernel of this EFIE has a severe singularity that makes it difficult to apply the method of moments [16]. However, the MPIE formulation involves a kernel having a weaker singularity [16], [17], [19]

$$
\begin{aligned}
& \mathbf{u}_{n} \times \sum_{i=1}^{N_{c}}\left\{j \omega \mathbf{A}_{i}(x, z)+\left(\nabla_{l}-\hat{\mathbf{y}} j \beta\right) \Phi_{i}(x, z)\right\}=0, \\
& (x, z) \in L_{j}\left(j=1, \cdots, N_{c}\right)
\end{aligned}
$$

where $\hat{\mathbf{u}}_{n}$ is an outward unit vector normal to the boundary $L_{j}$ of the $j$ th conductor, $\nabla_{l}$ is the transverse operator nabla, and $\mathbf{A}_{i}$ and $\Phi_{i}$ are the electrodynamic potentials generated by the free surface current on the $i$ th conductor. If the traditional Sommerfeld's formulation for the vector potential is used (formulation $\mathrm{C}$ in [23]), we can write

$$
\begin{aligned}
& \mathbf{A}_{i}(x, z)=\int_{L_{i}} \underline{\mathbf{K}}^{A}\left(x, z \mid x^{\prime}, z^{\prime}\right) \mathbf{J}\left(l^{\prime}\right) d l^{\prime} \\
& \Phi_{i}(x, z)=\int_{L_{i}} K^{\Phi}\left(x, z \mid x^{\prime}, z^{\prime}\right) \frac{j}{\omega}\left(\nabla_{l}^{\prime}-\hat{\mathbf{y}} j \beta\right) \mathbf{J}\left(l^{\prime}\right) d l^{\prime}
\end{aligned}
$$

where primed quantities stand for source coordinates. The form of the dyadic kernel $\underline{\mathbf{K}}^{A}$ is

$$
\underline{\mathbf{K}}^{A}=\left[\begin{array}{ccc}
K_{x x}^{A} & 0 & K_{x z}^{A} \\
0 & K_{x x}^{A} & K_{y z}^{A} \\
K_{z x}^{A} & K_{z y}^{A} & K_{z z}^{A}
\end{array}\right] .
$$

An alternative formulation reported in [23] gives rise to fewer nonzero terms in $\underline{K}^{A}$ provided the conductors are embedded in a single layer. Nevertheless, the use of such formulation does not add advantages in the frame of the approach used in this paper because the total number of functions to be approximated via complex images is the same. Thus, we adopt the classical formulation.

\section{A. Spectral-Domain Kernel}

It is well known that it is feasible to obtain closed-form expressions for $\underline{\mathbf{K}}^{A}$ and $K^{\Phi}$ in the spectral domain (namely, $\underline{\tilde{\mathbf{K}}}^{A}$ and $\tilde{K}^{\Phi}$ ) by using a transmission-line network analog of the layered medium [14], [24], [23]. For the problem treated here, it is convenient to write the kernel in the following form:

$$
\begin{aligned}
\tilde{K}^{\Phi}\left(k_{\rho} \mid z, z^{\prime}\right) & =\frac{j}{2 \omega \varepsilon_{0} u_{0}}\left[e^{-u_{0}\left|z-z^{\prime}\right|}+\tilde{F}^{\Phi}\left(k_{\rho}\right) e^{-u_{0}\left(z+z^{\prime}\right)}\right] \\
\tilde{K}_{y y}^{A}\left(k_{\rho} \mid z, z^{\prime}\right) & =\tilde{K}_{x x}^{A}\left(k_{\rho} \mid z, z^{\prime}\right) \\
& =\frac{j \omega \mu_{0}}{2 u_{0}}\left[e^{-u_{0}\left|z-z^{\prime}\right|}+\tilde{F}^{x x}\left(k_{\rho}\right) e^{-u_{0}\left(z+z^{\prime}\right)}\right] \\
\tilde{K}_{z z}^{A}\left(k_{\rho} \mid z, z^{\prime}\right) & =\frac{j \omega \mu_{0}}{2 u_{0}}\left[e^{-u_{0}\left|z-z^{\prime}\right|}+\tilde{F}^{z z}\left(k_{\rho}\right) e^{-u_{0}\left(z+z^{\prime}\right)}\right] \\
-\tilde{K}_{x z}^{A}\left(k_{\rho} \mid z, z^{\prime}\right) & =\tilde{K}_{z x}^{A}\left(k_{\rho} \mid z, z^{\prime}\right) \\
& =\frac{\omega \mu_{0}}{2 u_{0}} k_{x} \tilde{F}^{z x}\left(k_{\rho}\right) e^{-u_{0}\left(z+z^{\prime}\right)} \\
-\tilde{K}_{y z}^{A}\left(k_{\rho} \mid z, z^{\prime}\right) & =\tilde{K}_{z y}^{A}\left(k_{\rho} \mid z, z^{\prime}\right) \\
& =\frac{\beta}{k_{x}} \tilde{K}_{z x}^{A}\left(k_{\rho} \mid z, z^{\prime}\right)
\end{aligned}
$$

where $u_{0}=\sqrt{k_{\rho}^{2}-k_{0}^{2}}$ and $k_{\rho}^{2}=k_{x}^{2}+\beta^{2}\left(k_{x}\right.$ and $\beta$ are the Cartesian spectral variables, and $k_{\rho}$ the radial polar spectral variable). The functions $\tilde{F}^{i i}$ ( $i i$ stands for $\Phi, x x, z z$ or $z x$ ) have the following form:

$$
\begin{aligned}
\tilde{F}^{\Phi}\left(k_{\rho}\right) & =\frac{1}{k_{\rho}^{2}}\left(k_{0}^{2} \overleftarrow{\Gamma}^{h}+u_{0}^{2} \overleftarrow{\Gamma}^{e}\right) \\
\tilde{F}^{x x}\left(k_{\rho}\right) & =\overleftarrow{\Gamma}^{h}
\end{aligned}
$$




$$
\begin{aligned}
& \tilde{F}^{z z}\left(k_{\rho}\right)=\frac{u_{0}^{2}}{k_{\rho}^{2}} \overleftarrow{\Gamma}^{h}-\left(1+\frac{u_{0}^{2}}{k_{\rho}^{2}}\right) \overleftarrow{\Gamma}^{e} \\
& \tilde{F}^{z x}\left(k_{\rho}\right)=\frac{u_{0}}{k_{\rho}^{2}}\left(\overleftarrow{\Gamma}^{h}-\overleftarrow{\Gamma}^{e}\right) .
\end{aligned}
$$

$\overleftarrow{\Gamma}^{h}$ and $\overleftarrow{\Gamma}^{e}$ being the voltage reflection coefficient of the transmission-line analog, such as described in [23]. Superscripts $e$ and $h$ refers to TM and TE equivalent networks, respectively.

An important feature of the functions in (5)-(9) is the presence of branch points at $k_{\rho}= \pm k_{0}$. These branch points are related to the free-space unbounded upper layer of the structure. The Riemann surface $\operatorname{Re}\left\{u_{0}\right\} \geq 0$ of the complex $k_{\rho}$-plane must be chosen when computing $\underline{\mathbf{K}}^{A}$ and $K^{\Phi}$ in the space domain so as to avoid increasing exponential functions. The functions in (5)-(9) may also have a finite number of poles in the real axis of the $k_{\rho}$-plane. These poles represent the propagation constants of the surface-wave modes of the structure in the absence of sources. The $\tilde{F}^{i i}\left(k_{\rho}\right)$ functions in (10)-(13) exhibit the same complex plane topology. This latter point will play an important role in the development of our numerical approach.

\section{B. Space-Domain Kernel}

Once the spectral version of the integral-equation kernel is known, its 2-D space-domain counterpart can be obtained carrying out the following spectral integral (inverse Fourier transform):

$$
G\left(\left|x-x^{\prime}\right|, z \mid z^{\prime}\right)=\frac{1}{2 \pi} \int_{-\infty}^{\infty} e^{-j k_{x}\left|x-x^{\prime}\right|} \tilde{G}\left(k_{\rho} \mid z, z^{\prime}\right) d k_{x} .
$$

$G$ and $\tilde{G}$ being the generic spatial and spectral representations of $K^{\Phi}$ or any element of $\underline{\mathbf{K}}^{A}$. Straightforward numerical integration in (14) is not advisable due to the oscillatory and slowly decaying integrands. Special techniques must be used to perform such numerical integrations [17]. However, the integrands depend on $\beta$ in a complicated form. Therefore, such integrals should be evaluated for each guess value of the propagation constant in the eigenvalue search process.

In this paper, we describe an approach to perform the spectral integration in closed form. The method is based on the complex image technique. The complex image method has already been successfully used for the analysis of planar circuits, antennas, and scattering problems [20], [25]-[27]. The authors have recently adapted this technique to analyze guidance in strictly planar structures [21], [22]. In [21] and [22], the surface wave poles and the quasi-static behavior were removed from the spectral Green's functions, and the remaining part was approximated by using a finite number of images of the form $A\left(e^{\gamma u_{0}} / u_{0}\right)$, where $A$ and $\gamma$ were determined by means of the generalized pencil of function (GPoF) [28]. A closed-form expression for the space-domain Green's function was obtained in the form of a short expansion. Moreover, this expression was an explicit function of $\beta$, thus avoiding the need for successive image evaluations in the root-searching process. This approach cannot be applied to the problem treated here since the resulting approximated Green's functions do not include the explicit dependence on the $z$ and $z^{\prime}$ variables. Therefore, transmission lines having conductors with arbitrary cross section could not be analyzed in an efficient way using such a procedure. The solution proposed in this paper is to approximate only the $\tilde{F}^{i i}$ functions (10)-(13) as a sum of complex exponential functions, and not the whole Green's functions. Thus, the dependence on $z$ and $z^{\prime}$ of the Green's functions remains explicit. The underlying idea is to analytically perform the integration (14) by using the following identity [29]:

$$
\begin{aligned}
\frac{1}{2 \pi} \int_{-\infty}^{\infty} \frac{e^{-\gamma \sqrt{k_{x}^{2}+\alpha^{2}}}}{\sqrt{k_{x}^{2}+\alpha^{2}}} e^{-j k_{x}\left|x-x^{\prime}\right|} d k_{x} \\
=\frac{1}{\pi} K_{0}\left(\alpha \sqrt{\left(x-x^{\prime}\right)^{2}+\gamma^{2}}\right)
\end{aligned}
$$

where $K_{0}(\cdot)$ is the zeroth-order modified Bessel function of the second kind and $\alpha^{2}=\beta^{2}-k_{0}^{2}$ is supposed to be positive in the bound (nonleaky) regime. Expression (15) can be regarded as the 2-D Sommerfeld identity. This identity makes it possible to readily obtain the spatial version of the term with the $\left|z-z^{\prime}\right|$ dependence in the functions (5)-(7). The problem would be completely solved if we had a mean to analytically obtain the spatial-domain counterpart of the remaining of the spectral functions (5)-(7) and the functions (8) and (9). In order to simplify the formulation, these functions can be represented by a generalized spectral function having the following form:

$$
\tilde{S}^{i i}\left(k_{\rho} \mid z, z^{\prime}\right)=C^{i i} \tilde{F}^{i i}\left(k_{\rho}\right) \frac{e^{-u_{0}\left(z+z^{\prime}\right)}}{u_{0}}
$$

where the superscript $i i$ take the values $\Phi, x x, z z$ or $z x$, whereas $C^{i i}$ is a frequency-dependent parameter whose form depends on the value of this superscript.

It should be noted that the generalized spectral function (16) does not actually represent the $\tilde{K}_{z x}^{A}$ (8) element of $\underline{\tilde{\mathbf{K}}}^{A}$ due to the explicit presence of a $k_{x}$ factor in this term. However, it is evident from (14) that the spatial version of $\tilde{K}_{z x}^{A}$ can be obtained by deriving respect to $\left|x-x^{\prime}\right|$ the spatial version of $\tilde{K}_{z x}^{A} / k_{x}$. The latter expression does fit the form of the generalized spectral function (16). In this way, the problem reduces to obtain the spatial counterpart of (16) in a efficient way.

In principle, (15) could also be used to perform the integration (14) for $\tilde{S}^{i i}$ once we have approximated the $\tilde{F}^{i i}$ functions with complex images. However, before proceeding in this manner, it is necessary to extract out from $\tilde{F}^{i i}$ their asymptotic behavior for $k_{x} \gg k_{0}$ and the contribution of the surface-wave poles so that the complex images can properly approximate the functions [22], [27]. This poses a problem since the calculation of the spectral integral for the surface-wave terms cannot be carried out by using (15) or the approach in [22]. In this paper, an efficient procedure is presented to overcome this difficulty.

By applying the outlined method, $\tilde{F}^{i i}\left(k_{\rho}\right)$ in (16) can be expressed in the following approximated form:

$$
\tilde{F}^{i i}\left(k_{\rho}\right) \approx \tilde{F}_{I}^{i i}\left(k_{\rho}\right)+\tilde{F}_{p}^{i i}\left(k_{\rho}\right)+F_{0}^{i i}
$$

where $F_{0}^{i i}$ are the large argument $\left(k_{x} \gg k_{0}\right)$ approximation of $\tilde{F}^{i i}, \tilde{F}_{p}^{i i}$ are the surface-wave contributions, and $\tilde{F}_{I}^{i i}$ are the parts of the $\tilde{F}^{i i}$ functions to be approximated with complex images. Using this procedure, $\widetilde{S}^{i i}$ can be written as

$$
\tilde{S}^{i i}\left(k_{\rho} \mid z, z^{\prime}\right) \approx \tilde{S}_{I}^{i i}+\tilde{S}_{p}^{i i}+\tilde{S}_{0}^{i i} .
$$


In the following, these terms will be described and their contributions to the spatial-domain kernel will be computed.

1) Asymptotic Contribution: When the asymptotic limit of $\tilde{F}^{i i}$ for $k_{x} \rightarrow \infty$ is different from zero, there are problems in the direct application of complex image technique for the approximation of $\tilde{F}^{i i}$ since the complex image terms tend to zero when $k_{x} \rightarrow \infty$. In that case, the asymptotic limit of $\tilde{F}^{i i}$, which have been referred to as $F_{0}^{i i}$, should be always extracted out from $\tilde{F}^{i i}$ before applying the complex image scheme. By taking the limit $k_{x} \rightarrow \infty$ in the $\tilde{F}^{i i}\left(k_{\rho}\right)$ functions, we get

$$
F_{0}^{\phi}=\kappa \quad F_{0}^{x x}=0 \quad F_{0}^{z z}=2 \kappa \quad F_{0}^{z x}=0
$$

where

$$
\kappa=\frac{1-\sqrt{\varepsilon_{t N_{l}} \varepsilon_{z N_{l}}}}{1+\sqrt{\varepsilon_{t N_{l}} \varepsilon_{z N_{l}}}}
$$

$\varepsilon_{t N_{l}}$ and $\varepsilon_{z N_{l}}$ being the relative permittivities of the $N_{l}$ th layer in directions perpendicular and parallel to the $z$-axis, respectively. The form of the spectral $S_{0}^{i i}$ term is then

$$
\tilde{S}_{0}^{i i}\left(k_{\rho} \mid z, z^{\prime}\right)=C^{i i} F_{0}^{i i} \frac{e^{-u_{0}\left(z+z^{\prime}\right)}}{u_{0}} .
$$

This function can be easily transformed into the spatial domain by using (15), thus obtaining

$$
S_{0}^{i i}\left(x, z \mid x^{\prime}, z^{\prime}\right)=C^{i i} F_{0}^{i i} K_{0}\left(\alpha \sqrt{\left(x-x^{\prime}\right)^{2}+\left(z+z^{\prime}\right)^{2}}\right) .
$$

2) Surface-Wave Term: The complex exponential functions are not suitable to reproduce the decaying behavior associated to the poles of the Green's functions. It is then advisable to remove these poles before obtaining the complex images, at least when the frequency is high enough to make their influence nonnegligible. The poles appearing in the spectral Green's functions are also present in the $\tilde{F}^{i i}\left(k_{\rho}\right)$ functions.

The first step to remove the influence of these poles is to locate them for each frequency value. This could be done by searching for the zeros of the transverse resonant conditions: $\overleftarrow{Z}^{e}+\vec{Z}^{e}=0$ (TM modes) and $\overleftarrow{Z}^{h}+\vec{Z}^{h}=0$ (TE modes). In these expressions, $\bar{Z}$ and $\vec{Z}$ are the input impedances looking to the left- and right-hand side, respectively, from an arbitrary point inside the layered medium. However, searching for the roots of the transverse resonant conditions is not a simple task, since a pole of these expressions may be very close to a zero, thus making the numerical search difficult. We have then employed an alternative method described in [30]: the location of the poles is obtained by performing a root search over a specific analytic complex function having no poles or branch points.

Once the poles are located, we have to extract out its contribution to the spectral function. Since the spectral functions are even functions of $k_{\rho}$, the poles always appear in pairs, and we can write for the generalized $\tilde{S}_{p}^{i i}\left(k_{\rho} \mid z, z^{\prime}\right)$ function

$$
\tilde{S}_{p}^{i i}\left(k_{\rho} \mid z, z^{\prime}\right)=C^{i i} \frac{e^{-u_{0}\left(z+z^{\prime}\right)}}{u_{0}} \sum_{p=1}^{N_{0}^{i i}} \frac{2 R_{p}^{i i} k_{\rho p}}{k_{\rho}^{2}-k_{\rho p}^{2}}
$$

where $N_{0}^{i i}$ is the number of poles in the function $\tilde{F}^{i i}, k_{\rho p}$ is the location of the $p$ th pole in the $k_{\rho}$-plane, and $R_{p}^{i i}$ is the residue of the $\tilde{F}^{i i}$ function at the pole $k_{\rho p}$. The residue $R_{p}^{i i}$ can be calculated by using the Cauchy's theorem

$$
R_{p}^{i i}=\frac{1}{2 \pi j} \oint \tilde{F}^{i i}\left(k_{\rho}\right) d k_{\rho}
$$

where the contour integral in (22) follows a path enclosing the pole at $k_{\rho p}$. This integral can be easily performed by using Gauss quadratures. Five or ten quadrature points are enough for our purposes. It is worth noting that this residue calculation has been carried out in the $k_{\rho}$-plane. Consequently, the $R_{p}^{i i}$ value is valid, for a given frequency, throughout the entire root search process. As a consequence, the CPU time consumption of this step is not important for the overall procedure.

The spatial counterpart of (21) is then obtained by proper spectral integration on $k_{x}(14)$

$$
S_{p}^{i i}\left(x, z \mid x^{\prime}, z^{\prime}\right)=C^{i i} \sum_{p=1}^{N_{0}^{i i}} 2 R_{p}^{i i} k_{\rho p} I_{p}\left(x, z \mid x^{\prime}, z^{\prime}\right) .
$$

Therefore, the problem reduces to the computation of the integral $I_{p}$

$$
I_{p}=\int_{-\infty}^{\infty} \frac{e^{-j k_{x}\left|x-x^{\prime}\right|}}{k_{x}^{2}+\delta_{p}^{2}} \frac{e^{-u_{0}\left(z+z^{\prime}\right)}}{u_{0}} d k_{x}
$$

where $\delta_{p}^{2}=\beta^{2}-k_{\rho p}^{2}$ is supposed to be positive in the bound (nonleaky) regime. Parseval's theorem allows us to rewrite (24) in the following form:

$$
I_{p}=\frac{1}{\delta_{p}} \int_{-\infty}^{\infty} K_{0}\left(\alpha \sqrt{t^{2}+\zeta^{2}}\right) e^{-\delta_{p}|t-X|} d t
$$

where $\alpha^{2}=\beta^{2}-k_{0}^{2}, \zeta=\left(z+z^{\prime}\right)$, and $X=\left|x-x^{\prime}\right|$. As far as the authors know, no closed-form solution is available for (25). Fortunately, fast numerical evaluation is possible once the logarithmic singularity of $K_{0}(\cdot)$ is extracted out. The singular part of the integral is analytically evaluated, whereas the regular part is amenable to be calculated by using low-order Laguerre quadratures (see Appendix A).

It should be pointed out that (24) only depends on the location of the pole and, therefore, it is the same for all the spectral functions (5)-(9), except for $\tilde{K}_{z x}^{A}$. To evaluate the contribution of this term, it is necessary to derive $I_{p}(24)$ with respect to $\left|x-x^{\prime}\right|$. The detailed evaluation of $I_{p}$ and its derivative with respect to $\left|x-x^{\prime}\right|, I_{p}^{\prime}$ is treated in Appendixes A and B.

3) Complex Images Term: Once the asymptotic and surface-wave terms of the spectral functions have been removed, a finite sum of complex images can be used to approximate the remaining part. The images are computed by means of the GPoF method [28]

$$
\tilde{F}_{I}^{i i}\left(k_{\rho}\right)=\tilde{F}^{i i}\left(k_{\rho}\right)-\tilde{F}_{p}^{i i}\left(k_{\rho}\right)-F_{0}^{i i} \approx \sum_{j=1}^{N_{m}^{i i}} a_{j}^{i i} e^{-\gamma_{j}^{i i} u_{0}}
$$


where $N_{m}^{i i}$ is the number of complex images employed to approximate $\tilde{F}_{I}^{i i}$. The approximated $\tilde{F}_{I}^{i i}$ give rise to the following approximated $\tilde{S}_{I}^{i i}$ functions:

$$
\tilde{S}_{I}^{i i}\left(k_{\rho} \mid z, z^{\prime}\right) \approx C^{i i} \sum_{j=1}^{N_{m}^{i i}} a_{j}^{i i} \frac{e^{-\left(z+z^{\prime}+\gamma_{j}^{i i}\right) u_{0}}}{u_{0}} .
$$

As a final result, the remaining spectral Green's functions have been expanded into a sum of terms representing cylindrical waves in the spectral domain. These are the type of functions obtained if we analyze our problem after substituting the dielectric layers for vacuum. Therefore, it is physically expected that just a few images will suffice to provide an excellent approximation of the desired spectral function. Using (15), we can write for the space-domain version of (27)

$$
\begin{aligned}
& S_{I}^{i i}\left(x, z \mid x^{\prime}, z^{\prime}\right) \\
& \quad \approx C^{i i} \sum_{j=1}^{N_{m}^{i i}} a_{j}^{i i} K_{0}\left(\alpha \sqrt{\left(x-x^{\prime}\right)^{2}+\left(z+z^{\prime}+\gamma_{j}^{i j}\right)^{2}}\right) .
\end{aligned}
$$

In order to apply the GPoF for obtaining $a_{j}^{i i}$ and $\gamma_{j}^{i i}$ in (26), we have sampled the spectral functions along a path in the $k_{\rho}$-plane, which is similar to that suggested in [31] for approximating three-dimensional (3-D) Green's functions. When using this approximation in our 2-D problem, the path in the $k_{\rho}$-plane maps into different paths in the $k_{x}$-plane as $\beta$ changes in the root searching process. These latter paths do not coincide with the real axis of the $k_{x}$-plane. According to (14), the value of $\tilde{G}\left(k_{\rho} \mid z, z^{\prime}\right)$-and, therefore, the value of $\tilde{F}_{I}^{i i}\left(k_{\rho}\right)$-is required along the real axis of the $k_{x}$-plane. It may be thought that our procedure is not correct because the approximating function in the $k_{x}$-plane has been obtained along a different path on that plane. However, since the spectral functions $\tilde{F}_{I}^{i i}\left(k_{\rho}\right)$ have no poles, analytic continuation can be invoked to justify that these functions are also valid along the real axis of the $k_{x}$-plane. This issue has been numerically verified and accurate results have been obtained for the approximation of $\tilde{F}_{I}^{i i}\left(k_{\rho}\right)$ along the $k_{x}$-plane real axis for many different values of $\beta$. Since a single set of complex images is used in the approximation of $\tilde{F}_{I}^{i i}\left(k_{\rho}\right)$ for any value of $\beta$, important CPU time savings are achieved in the root search process necessary for the determination of the propagation constants. This is an additional and essential advantage of our approach.

A crucial point that makes the approximation of the spectral functions work properly is that both $\tilde{F}_{I}^{i i}\left(k_{\rho}\right)$ and the complex image terms present the same branch points at $k_{\rho}= \pm k_{0}$. The use of different branch points in the complex image terms and in the spectral functions to be approximated leads to very poor quality approximations, as explained in [27].

Also, note that there are reasons to think that the complex images are particularly suitable to accurately approximate the spectral functions, since they give rise to terms representing cylindrical waves in the spectral domain. This is the kind of functions expected if we consider that the influence of the surface wave has been separately treated.

\section{Solving the Integral Equation}

Once the space-domain kernel is known, the method of moments is used to solve for the integral equation. The cross-sectional contours of the conductors have been approximated by piecewise linear segments, and the unknown currents have been expressed as an expansion into a set of basis functions whose coefficients have to be determined. Pulse functions have been used to approximate the axial component of the surface current density ( $y$-component) and triangle functions for the transverse components ( $x$ and $z$ components). The reader is referred to [14] to find a detailed formulation of the application of the method of moments with this kind of basis functions. It is worth mentioning that we have used a nonuniform distribution of the basis functions in each face of the conductors. The basis functions have been concentrated near the edges in order to reproduce in a better way the fast variations of the current density in those regions.

The application of the method of moments implies the integration of the kernel of the integral equation over straight segments on the cross section of the conductors (reaction integrals). To improve the efficiency and accuracy of the method, the logarithmic singularity of the kernel has been separately treated and its contribution analytically obtained. The remaining regular contribution to the reaction integrals has been evaluated with very low computational effort: no more than two or three Gauss-Legendre quadrature points have been found to be necessary for every integral.

\section{NUMERICAL RESULTS}

The performance of our approach will be primarily determined by the quality of the approximation of the kernel of the integral equation. In order to investigate this issue, let us focus our attention on a simple dielectric configuration involving all the elements of the reported method: a single dielectric slab over a ground plane. We are first interested in showing the accuracy of our method and the convenience of removing pole contributions before applying the GPoF. Consider a slab thickness of $h=0.635 \mathrm{~mm}$ and a relative dielectric constant of $\varepsilon_{r}=9.8$. The source point is located at $z^{\prime}=0$ (i.e., at the dielectric interface) and the field point at $z=0.3 \mathrm{~mm}$. We have computed the elements of the kernel in the space domain for the frequencies $f=10 \mathrm{GHz}$ and $f=30 \mathrm{GHz}$. At such frequencies, the spectral-domain kernel has a surface wave $\mathrm{TM}_{0}$ pole at $k_{\rho} \approx 1.007 k_{0}$ and $k_{\rho} \approx 1.2 k_{0}$, respectively. In Fig. 2, we compare the direct numerical integration method (which combines the Romberg and the weighted averages techniques [17]) against the method in this paper. The solid line of Fig. 2(a) shows $\left|K^{\Phi}\left(\left|x-x^{\prime}\right|, z, z^{\prime}\right)\right|$ for $f=10 \mathrm{GHz}$ as a function of $\left|x-x^{\prime}\right|$ (direct integration results). The relative difference between these data and the values computed with our approach (using eight complex images) is also depicted in that figure. We have included the discrepancy existing between numerical and quasi-analytical data both, when the surface wave pole is removed before computing the complex images and when it is not removed. We can see that the quality of the approximation is slightly better if the pole is removed. The impact of extracting the pole increases with $\left|x-x^{\prime}\right|$ because the dominant far field 


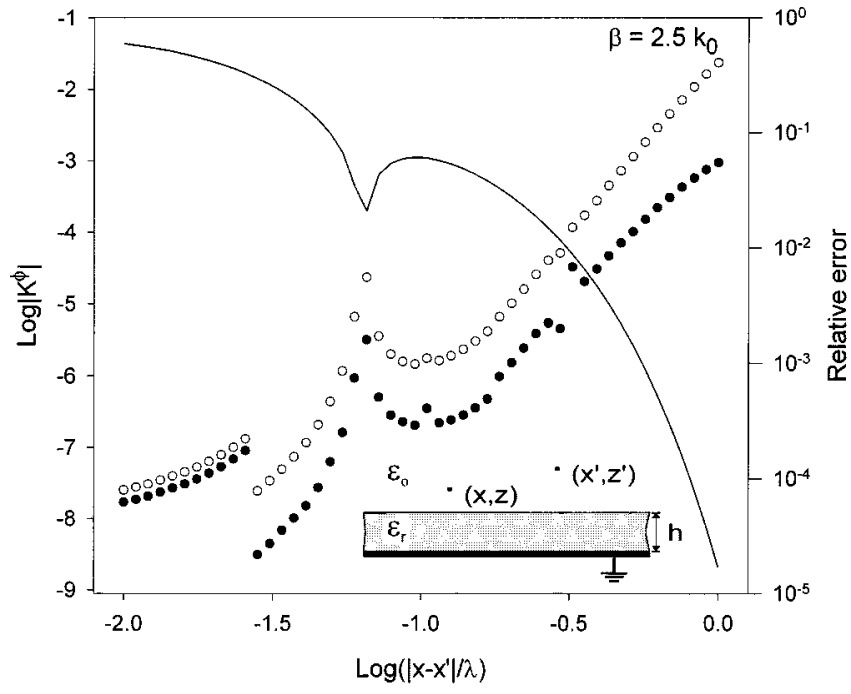

(a)

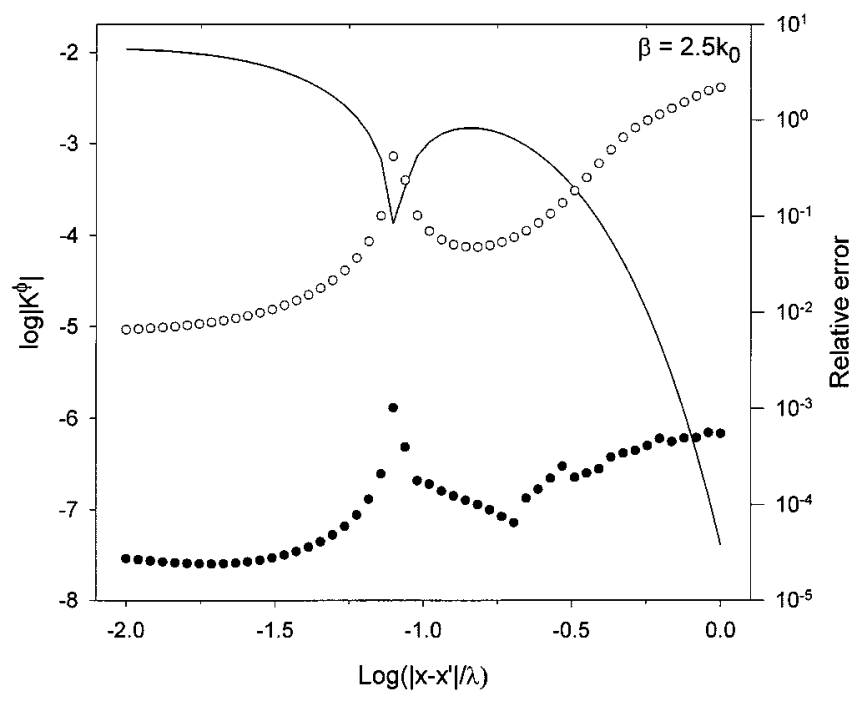

(b)

Fig. 2. Magnitude of $K^{\phi}$ (solid line) and relative difference between numerical integration computation and complex images computation with (black dots) and without pole extraction (white dots) for: (a) $10 \mathrm{GHz}$ and (b) $30 \mathrm{GHz}$. Data: $h=0.635 \mathrm{~mm}, \varepsilon_{r}=9.8, z=0$, and $z^{\prime}=0.3 \mathrm{~mm}$.

associated to the surface waves cannot be properly reproduced only by the complex images. Anyway, both approaches provide an acceptable error level all over the range of distances for which the approximated function is not negligible. However, for higher frequencies, the situation dramatically changes. Fig. 2(b) shows the same curves that in Fig. 2(a), but for a different frequency $f=30 \mathrm{GHz}$. In this case, it is obvious that the pole extraction is essential to keep the error below a reasonable level. The poor results obtained when poles are not extracted affects to the whole range of source-field point distances. Although we have only shown results for $K^{\Phi}$, the same conclusions can be applied to all the elements of the kernel of our integral equation, as the authors have explicitly verified from many numerical tests. We have also repeated these tests for different values of $z$ and $z^{\prime}$ and for more complex dielectric configurations. The conclusions are identical.

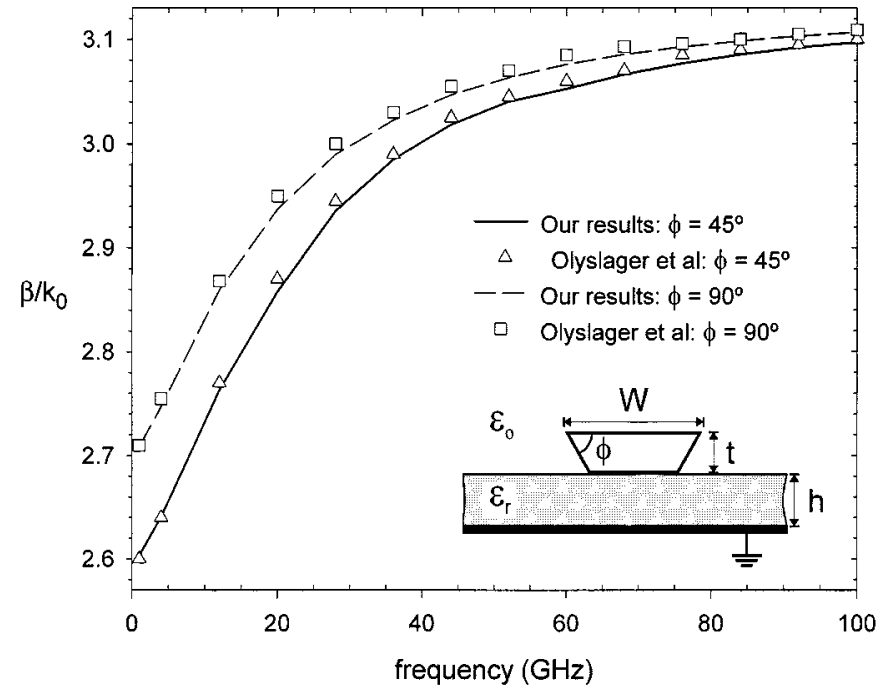

Fig. 3. Dispersion curve of the fundamental mode of the thick microstrip structure of the figure. Two different conductor cross section are considered. ( $\left.h=0.635 \mathrm{~mm}, w=3 \mathrm{~mm}, t=0.3 \mathrm{~mm}, \varepsilon_{r}=9.8\right)$.

As a first example of the application of the code based on the theory in this paper, we have analyzed the structure depicted in Fig. 3, i.e., a thick microstrip printed over the substrate used in the example in Fig. 2. The thickness of the conductor is $t=0.3 \mathrm{~mm}$. Fig. 3 shows the dispersion curves of the fundamental mode of this microstrip structure when the conductor is considered rectangular $\left(\phi=90^{\circ}\right)$ and trapezoidal $\left(\phi=45^{\circ}\right)$. Results for this structure have been previously published in [10] (for a narrower range of frequencies) and [15]. The former makes use of a space-domain MPIE formulation, but the spectral integrals are numerically computed. The latter uses a boundary integral-equation technique in conjunction with the method of moments. The results provided in [15] are included in Fig. 3 (they are very close to those reported in [10]). The agreement with our results is very good. In [10], results are also presented for the particular case $t=0$ $\mathrm{mm}$ (infinitely thin microstrip). The fundamental and higher modes in the bound regime for such case have been also reproduced with our code.

A structure printed on the same substrate and containing two coupled thick strips has been also analyzed in [15]. This structure is shown in Fig. 4. The dispersion curves for the propagation constants of the even and odd modes are plotted there. The data reported in [15] are also included for comparison and excellent agreement has been found. Axial and transverse components of the current densities have been modeled by ten pulse and triangle functions, respectively, over each conductor.

It is worth mentioning that the results presented in Figs. 3 and 4 can be obtained only if the surface-wave pole contribution is removed before using complex images. Otherwise, results become unstable when frequency increases, since the surface wave poles contribution becomes important. In the particular example studied here, this occurs when $f>30 \mathrm{GHz}$ for the first surface wave pole $\left(\mathrm{TM}_{0}\right)$ and when $f>48 \mathrm{GHz}$ for the second pole $\left(\mathrm{TE}_{1}\right)$. Subsequent poles are important above $100 \mathrm{GHz}$.

To illustrate the performance of the method when dealing with wire transmission lines, the results reported in [15] for 


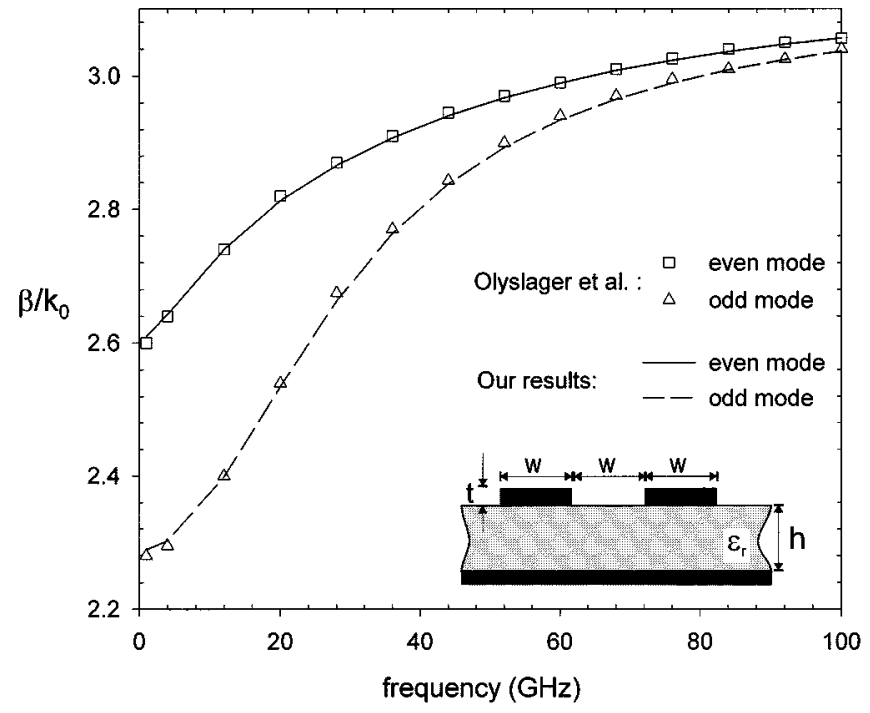

Fig. 4. Propagation constant as a function of frequency for the even and odd mode of two coupled microstrips. Data: $h=0.635 \mathrm{~mm}, w=1 \mathrm{~mm}, t=0.3$ $\mathrm{mm}, \varepsilon_{r}=9.8$

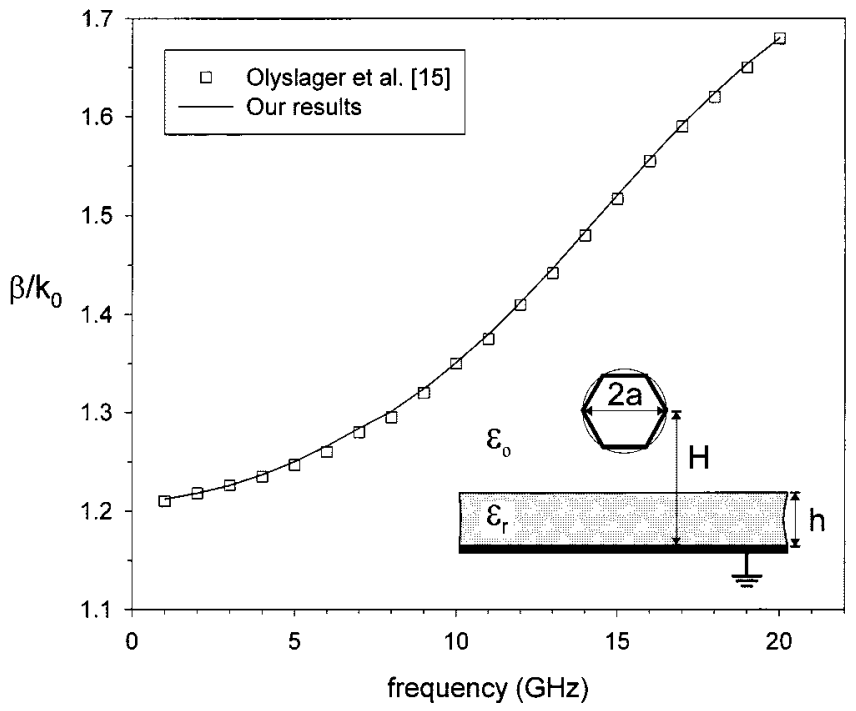

Fig. 5. Dispersion curve for the fundamental mode of the structure in the figure. Data: $h=3 \mathrm{~mm}, a=0.75 \mathrm{~mm}, \varepsilon_{r}=4, H=4.5 \mathrm{~mm}$

the fundamental mode of the wire transmission line depicted in Fig. 5 have been reproduced with our code. The circular conductor has been approximated by an hexagon with the same area. The axial component of the surface current density has been modeled with ten pulse functions. Results are plotted in Fig. 5 along with those reported in [15]. An excellent agreement is observed once again.

As a final example, let us consider the three-conductor transmission line studied by Hsu et al. in [14] by using the MPIE scheme with numerical evaluation of the spectral integrals [14, Fig. 8]. Three different conductor cross sections are considered: trapezoidal, rectangular, and inverted trapezoidal. Fig. 6 shows the dispersion curves of the three fundamental modes supported by each three conductor system. Twelve basis functions over each conductor have been used to obtain those results. Fig. 6 also includes the results reported in [14], which agree very satisfacto-

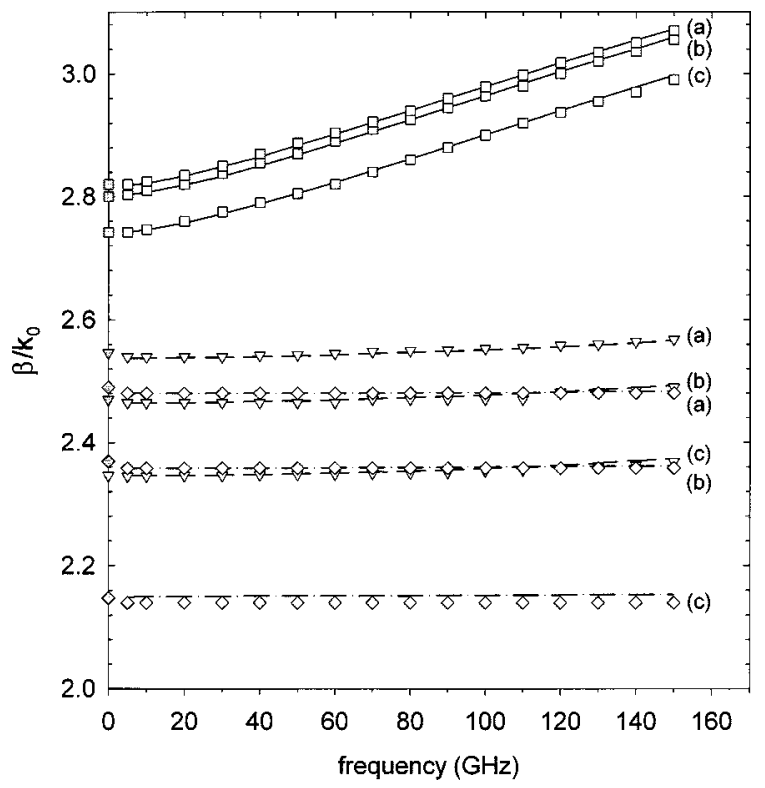

Fig. 6. Dispersion curves of the three fundamental modes of the transmission-line configurations (a)-(c) in [14, Fig. 8]. Symbols for every configuration: our results for mode 1: solid line; results from [14]: white squares. Our results for mode 2: dashed line; results from [14]: white triangles. Our result for mode 3: dots and dashes; results from [14]: white diamonds. Quasi-static results obtained with the method reported in [3] are also included: mode 1: grey squares; mode 2: grey triangles; mode 3: grey diamonds.

rily with ours. The low-frequency limit of the dispersion curves have been checked against the results obtained by a highly accurate quasi-TEM code [3] with very good agreement.

Finally, let us write a few words about CPU time consumption. As it has been mentioned in this section, an ad hoc numerical integration scheme for obtaining the space-domain kernel of the MPIE has been programmed so as to check the proposed method. Although the numerical scheme takes into account the special features of the integrands, for a given level of accuracy, CPU times are always much shorter if the approach in this paper is used. CPU time saving with respect numerical integration is more relevant when the structure becomes more and more complex. For the examples studied here, a reduction factor in CPU time above ten has been found for all the cases.

\section{CONCLUSIONS}

In this paper, the complex image method, originally intended for 3-D problems, has been successfully adapted to handle the guidance problem of open transmission lines with arbitrary cross-sectional conductors on a layered substrate. It has been proven that the correct performance of this technique in all the range of frequencies requires to treat separately the surface-wave contribution of the kernel of the integral equation. An efficient method has been presented to take into account this surface-wave contribution. These ideas have been implemented into a MPIE formulation to solve for the multiconductor problem. Nonuniform discretization is incorporated so as to minimize the size of the matrix of moments. The calculation of each element of this matrix has been also speeded up thanks to a quasi-analytical evaluation of the reaction integrals. The computer code based on this theory has been conveniently checked against canonical examples published on the literature 
and against results provided by a computer code based on the computation of the MPIE kernel by direct numerical integration. This program has made it possible to verify the relative accuracy and numerical efficiency of the proposed approach.

\section{APPENDIX A}

We are faced with the problem of calculating $I_{p}$ in (25). This integral turns out to be the convolution of a exponential function with a zeroth-order modified Bessel function of the second kind. The modified Bessel function has a logarithmic singularity in the origin that should be removed as follows:

$$
I_{p}=I_{a}-I_{b}
$$

where

$$
\begin{aligned}
& I_{a}=\frac{1}{\delta_{p}} \int_{-\infty}^{\infty}[ K_{0}\left(\alpha \sqrt{t^{2}+\zeta^{2}}\right) \\
&\left.+\log \left(\alpha \sqrt{t^{2}+\zeta^{2}}\right)\right] e^{-\delta_{p}|t-X|} d t \\
& I_{b}=\frac{1}{\delta_{p}} \int_{-\infty}^{\infty} \log \left(\alpha \sqrt{t^{2}+\zeta^{2}}\right) e^{-\delta_{p}|t-X|} d t .
\end{aligned}
$$

The integrand in (31) includes the singularity of $K_{0}(\cdot)$ and, therefore, it is advisable to avoid the numerical evaluation of that integral. Splitting the integral $I_{b}$ into two terms to eliminate the module in the argument of the exponential function and changing variables, $I_{b}$ can be rewritten as

$$
\begin{aligned}
I_{b}=\frac{1}{\delta_{p}} & \int_{0}^{\infty} \log \left(\alpha \sqrt{(u-X)^{2}+\zeta^{2}}\right) e^{-\delta_{p} u} d u \\
& +\frac{1}{\delta_{p}} \int_{0}^{\infty} \log \left(\alpha \sqrt{(u+X)^{2}+\zeta^{2}}\right) e^{-\delta_{p} u} d u .
\end{aligned}
$$

If use is made of the following result [29]:

$$
\int_{0}^{\infty} \log (x+b) e^{-\delta_{p} x} d x=\frac{1}{\delta_{p}}\left\{\log b-e^{\delta_{p} b} E_{i}\left(-\delta_{p} b\right)\right\}
$$

where $E_{i}$ is the exponential integral function, the integral $I_{b}$ can be written in the following form:

$$
\begin{aligned}
& I_{b}=\frac{1}{2 \delta_{p}^{2}}[ 2 \log \left(\alpha^{2}\left(X^{2}+\zeta^{2}\right)\right)-e^{-\delta_{p} X} \\
& \cdot\left[e^{j \zeta \delta_{p}} E_{i}\left(\delta_{p}(X-j \zeta)\right)+e^{-j \zeta \delta_{p}} E_{i}\left(\delta_{p}(X+j \zeta)\right)\right] \\
&-e^{\delta_{p} X}\left[e^{j \zeta \delta_{p}} E_{i}\left(\delta_{p}(-X-j \zeta)\right)\right. \\
&\left.+e^{-j \zeta \delta_{p}} E_{i}\left(\delta_{p}(-X+j \zeta)\right)\right]
\end{aligned}
$$

Assuming that $\delta_{p}$ is a real variable (this is true only if we are searching for real values of the propagation constant), we can obtain a simpler expression for $I_{b}$

$$
\begin{aligned}
I_{b}=\frac{1}{\delta_{p}^{2}}[ & \log \left(\alpha^{2}\left(X^{2}+\zeta^{2}\right)\right) \\
& -e^{-\delta_{p} X} \operatorname{Re}\left\{e^{j \zeta \delta_{p}} E_{i}\left(\delta_{p}(X-j \zeta)\right)\right\} \\
& \left.-e^{\delta_{p} X} \operatorname{Re}\left\{e^{j \zeta \delta_{p}} E_{i}\left(\delta_{p}(-X-j \zeta)\right)\right\}\right] .
\end{aligned}
$$

In this way, $I_{b}$ in (31) is evaluated in closed form. In order to calculate the integral $I_{a}$, we express this integrand in a form amenable to apply Laguerre quadratures

$$
\begin{aligned}
I_{a} & =\frac{1}{\delta_{p}} \int_{-\infty}^{\infty} f(t) e^{-\delta_{p}|t-X|} \\
& =\frac{1}{\delta_{p}^{2}}\left\{\int_{0}^{\infty} f\left(X-\frac{u}{\delta_{p}}\right) e^{-u} d u+\int_{0}^{\infty} f\left(X+\frac{u}{\delta_{p}}\right) e^{-u} d u\right\}
\end{aligned}
$$

where

$$
f(t)=K_{0}\left(\alpha \sqrt{t^{2}+\zeta^{2}}\right)+\log \left(\alpha \sqrt{t^{2}+\zeta^{2}}\right) .
$$

Since the integrand in (36) is smooth, only five or ten Laguerre quadrature points suffice to obtain accurate results for $I_{a}$. Summing up, the $I_{p}$ integral can be calculated in a efficient quasiclosed form.

\section{APPENDIX B}

Here, the evaluation of the following integral:

$$
I_{p}^{\prime}=\int_{-\infty}^{\infty} k_{x} \frac{e^{-j k_{x}\left|x-x^{\prime}\right|}}{k_{x}^{2}+\delta_{p}^{2}} \frac{e^{-u_{0}\left(z+z^{\prime}\right)}}{u_{0}} d k_{x}
$$

is explained. This integral is necessary for the computation of $K_{x z}^{A}\left(\left|x-x^{\prime}\right|, z \mid z^{\prime}\right)$. From (24), it is clear that $I_{p}^{\prime}$ can be expressed as $I_{p}^{\prime}=j\left(d I_{p}\right) /(d X)$, where $X=\left|x-x^{\prime}\right|$. To perform this derivation over the convolution form of $I_{p}(25)$, the integrand must be split into two terms to avoid the presence of the module in the argument of the exponential function

$$
\begin{aligned}
& I_{p}=\frac{1}{\delta_{p}} \int_{-\infty}^{X} K_{0}\left(\alpha \sqrt{t^{2}+\zeta^{2}}\right) e^{-\delta_{p}(X-t)} d t \\
& \quad+\frac{1}{\delta_{p}} \int_{X}^{\infty} K_{0}\left(\alpha \sqrt{t^{2}+\zeta^{2}}\right) e^{-\delta_{p}(t-X)} d t
\end{aligned}
$$

$I_{p}^{\prime}$ can now be calculated by deriving the expression above

$$
\begin{aligned}
I_{p}^{\prime}=-j \int_{-\infty}^{X} & K_{0}\left(\alpha \sqrt{t^{2}+\gamma^{2}}\right) e^{-\delta_{p}(X-t)} d t \\
& +j \int_{X}^{\infty} K_{0}\left(\alpha \sqrt{t^{2}+\zeta^{2}}\right) e^{-\delta_{p}(t-X)} d t .
\end{aligned}
$$

The integral $I_{p}^{\prime}(40)$ is split into two integrals having the same form as those in (39). This makes it possible to use the results obtained in Appendix A for $I_{p}$ to calculate $I_{p}^{\prime}$. Extracting out again the logarithmic singularity of the modified Bessel function, we can write

$$
I_{p}^{\prime}=I_{a}^{\prime}-I_{b}^{\prime}
$$

where

$$
\begin{gathered}
I_{b}^{\prime}=\frac{j}{2 \delta_{p}}\left[e^{-\delta_{p} X}\left[e^{j \zeta \delta_{p}} E_{i}\left(\delta_{p}(X-j \zeta)\right)+e^{-j \zeta \delta_{p}} E_{i}\left(\delta_{p}(X+j \zeta)\right)\right]\right. \\
-e^{\delta_{p} X}\left[e^{j \zeta \delta_{p}} E_{i}\left(\delta_{p}(-X-j \zeta)\right)\right. \\
\left.\left.+e^{-j \zeta \delta_{p}} E_{i}\left(\delta_{p}(-X+j \zeta)\right)\right]\right]
\end{gathered}
$$

Note that $I_{b}^{\prime}(42)$ does not require new numeric calculation of the exponential and exponential integral functions since the terms already calculated in (34) can be easily reused here. In a similar 
way, a new application of the Laguerre quadratures is not necessary to calculate $I_{a}^{\prime}$ since $I_{a}^{\prime}$ is split into the same two integrals appearing in the computation of $I_{a}$. Hence, no additional computation effort is necessary to perform the integration (38) once (25) has been calculated.

\section{ACKNOWLEDGMENT}

While preparing this paper's manuscript, the authors were aware of the fact that Prof. K. A. Michalski, Texas A\&M University, College Station, had carried out some unpublished research on the application of complex image method to the analysis of transmission lines with arbitrary cross section in multilayered media [32]. This unpublished research was kindly supplied by Prof. K. A. Michalski. Although the approach followed in [32] differs to a certain extent from that followed in this paper, it provided further insight during the preparation of this paper's final manuscript. Therefore, the authors gratefully acknowledge Prof. K. A. Michalski for his generosity and help.

\section{REFERENCES}

[1] A. Deutsch et al., "When are transmission-line effects important for on-chip interconnections?," IEEE Trans. Microwave Theory Tech., vol. 45, pp. 1836-1846, Oct. 1997.

[2] N. Fache, F. Olyslager, and D. De Zutter, "Full-wave analysis of coupled perfectly conducting wires in a multilayered medium," IEEE Trans. Microwave Theory Tech., vol. 39, pp. 673-681, Apr. 1991.

[3] J. Bernal, F. Medina, and M. Horno, "Quick quasi-EM analysis of multiconductor transmission lines with rectangular cross section," IEEE Trans. Microwave Theory Tech., vol. 45, pp. 1619-1626, Sept. 1997.

[4] G. Cano, F. Medina, and M. Horno, "On the efficient implementation of SDA for boxed strip-like and slot-like structures," IEEE Trans. Microwave Theory Tech., vol. 46, pp. 1801-1806, Nov. 1998.

[5] M. S. Alam, M. Koshiba, K. Hirayama, and Y. Hayashi, "Hybrid-mode analysis of multilayered and multiconductor transmission lines," IEEE Trans. Microwave Theory Tech., vol. 45, pp. 205-211, Feb. 1997.

[6] C. J. Railton and J. P. McGeehan, "An analysis of microstrip with rectangular and trapezoidal conductor cross sections," IEEE Trans. Microwave Theory Tech., vol. 38, pp. 1017-1022, Aug. 1990.

[7] Z. Ma, E. Yamashita, and S. Xu, "Hybrid-mode analysis of planar transmission lines with arbitrary metallization cross sections," IEEE Trans. Microwave Theory Tech., vol. MTT-41, pp. 491-497, Mar. 1993.

[8] K. M. Rahman and C. Nguyen, "Full-wave analysis of coplanar strips considering the finite strip metallization thickness," IEEE Trans. Microwave Theory Tech., vol. 42, pp. 2177-2179, Aug. 1994.

[9] F. J. Schmckle and R. Pregla, "The method of lines for the analysis of planar waveguides with finite metallization thickness," IEEE Trans. Microwave Theory Tech., vol. 39, pp. 107-111, Jan. 1991.

[10] K. A. Michalski and D. Zheng, "Rigorous analysis of open microstrip lines of arbitrary cross section in bound and leaky regimes," IEEE Trans. Microwave Theory Tech., vol. 37, pp. 2005-2010, Dec. 1989.

[11] R. T. Kollipara and V. K. Tripathi, "Dispersion characteristics of moderately thick microstrip lines by the spectral domain method," IEEE Microwave Guided Wave Lett., vol. 2, pp. 100-101, Mar. 1992.

[12] T. Kitazawa, "Loss calculation of single and coupled strip lines by extended spectral domain approach," IEEE Microwave Guided Wave Lett., vol. 3, pp. 211-213, July 1993.

[13] J.-T. Kuo and T. Itoh, "Hybrid-mode computation of propagation and attenuation characteristics of parallel coupled microstrips with finite metallization thickness," IEEE Trans. Microwave Theory Tech., vol. 45, pp. 274-280, Feb. 1997.

[14] C.-I. G. Hsu, R. F. Harrington, K. A. Michalski, and D. Zheng, “Analysis of multiconductor transmission lines of arbitrary cross section in multilayered uniaxial media," IEEE Trans. Microwave Theory Tech., vol. 41, pp. 70-78, Jan. 1993.
[15] F. Olyslager, D. De Zutter, and K. Blomme, "Rigorous analysis of the propagation characteristic of general lossless and lossy multiconductor transmission lines in multilayered media," IEEE Trans. Microwave Theory Tech., vol. 41, pp. 79-88, Jan. 1993.

[16] A. W. Glisson and D. R. Wilton, "Simple and efficient numerical methods for problems of electromagnetic radiation and scattering from surfaces," IEEE Trans. Antennas Propagat., vol. AP-28, pp. 593-603, Sept. 1980.

[17] J. R. Mosig and F. E. Gardiol, "Analytic and numerical techniques in the Green's function treatment of microstrip antennas and scatterers," Proc. Inst. Elect. Eng., pt. H, vol. 130, pp. 175-182, Mar. 1983.

[18] K. A. Michalsky, "The mixed potential electric field integral equation for objects in a layered media," Arch. Elektron. Uebertrag., vol. 39, pp. 317-322, Sept.-Oct. 1985.

[19] J. R. Mosig, "Arbitrarily shaped microstrip structures and their analysis with a mixed potential integral equation," IEEE Trans. Microwave Theory Tech., vol. 36, pp. 314-323, Feb. 1988.

[20] D. G. Fang, J. J. Yang, and G. Y. Delisle, "Discrete image theory for horizontal electric dipoles in a multilayered medium," Proc. Inst. Elect. Eng., pt. H, vol. 135, pp. 297-303, Oct. 1988.

[21] J. Bernal, F. Medina, R. R. Boix, and M. Horno, "Full wave analysis of multilevel, multiconductor planar transmission lines using MPIE and complex images," in Proc. Asia-Pacific Microwave Conf., vol. 2, Yokohama, Japan, Dec. 8-11, 1998, pp. 417-420.

[22] _ - "Fast full wave analysis of multistrip transmission lines based on MPIE and complex images," IEEE Trans. Microwave Theory Tech., vol. 48, pp. 445-452, Mar. 2000.

[23] K. A. Michalski and D. Zheng, "Electromagnetic scattering and radiation by surfaces of arbitrary shape in layered media-Part I: Theory," IEEE Trans. Antennas Propagat., vol. 38, pp. 335-344, Mar. 1990.

[24] L. B. Felsen and N. Marcuvitz, Radiation and Scattering of Waves. Englewood Cliffs, NJ: Prentice-Hall, 1973.

[25] Y. L. Chow, J. J. Yang, D. G. Fang, and G. E. Howard, "A closed-form spatial Green's function for the thick microstrip substrate," IEEE Trans. Microwave Theory Tech., vol. 39, pp. 588-592, Mar. 1991.

[26] M. I. Aksun and R. Mittra, "Derivation of closed-form Green's functions for a general microstrip geometry," IEEE Trans. Microwave Theory Tech., vol. 40, pp. 2055-2061, Nov. 1992.

[27] R. A. Kipp and C. H. Chan, "Complex image method for sources in bounded regions of multilayer structures," IEEE Trans. Microwave Theory Tech., vol. 42, pp. 860-865, May 1994.

[28] T. K. Sarkar and O. Pereira, "Using the matrix pencil method to estimate the parameters of a sum of complex exponentials," Antennas Propagat. Mag., vol. 37, pp. 48-55, Feb. 1995.

[29] I. S. Gradshteyn and I. M. Ryzhik, Table of Integrals, Series, and Products. New York: Academic, 1980.

[30] F. Mesa and M. Horno, "Computation of proper and improper modes in multilayered bianisotropic waveguides," IEEE Trans. Microwave Theory Tech., vol. 43, pp. 233-235, Jan. 1995.

[31] M. I. Aksun, "A robust approach for the derivation of closed-form Green's functions," IEEE Trans. Microwave Theory Tech., vol. 44, pp. 651-658, May 1996.

[32] K. A. Michalski, private communication.

Joaquin Bernal, photograph and biography not available at time of publication.

Francisco Medina (M'90), photograph and biography not available at time of publication

Rafael R. Boix (M'97), photograph and biography not available at time of publication. 